\title{
SURFACES MINIMIZING AREA PLUS LENGTH OF SINGULAR CURVES
}

\author{
FRANK MORGAN
}

(Communicated by Peter Li)

\begin{abstract}
We give the first existence and regularity results on surfaces minimizing area plus length of singular curves, as in energy-minimizing interfaces in materials.
\end{abstract}

\section{INTRODUCTION}

Interfaces in materials are often modeled by area-minimizing surfaces, such as the soap film on tetrahedral frame of Figure 1.1(a) (on the next page). Such surfaces typically meet in threes at $120^{\circ}$ angles along singular curves. A better model might add a cost ("disclination energy") proportional to the length of the singular curves. [MT] suggests that such a minimizer of area plus length might look like the surface of Figure 1.1(b), with more area but a shorter singular curve. Note that four pieces of surface meet along the short curve in the center. Rough estimates suggest that this phenomenon would occur at a scale just below what can be detected by today's technology.

This paper gives the first mathematical results on the existence and structure of such energy-minimizing surfaces. The results apply to physical examples and provide a model for computer approximations (see Brakke [Br2]).

Earlier work by Cook [C] minimized area plus "length" for a single piece of surface with a free boundary "curve" constrained to lie in a plane, in higher dimensions. Recently we have discovered a number of the methods of our paper in excellent earlier work of Ecker [E] on the so-called thread problem, a problem in many ways more difficult, although it considers just a single surface (see $\S 5$ ). After completing this work, we found a conjecture by De Giorgi [DG, Congettura 2.7] on the existence of certain surfaces minimizing area plus length of singular curves.

More generally, there has been much work in various contexts on minimizing sums of energies of different dimensions, although typically codimensions 0 and 1. For example, work of Mumford and Shah [MuS] helps a computer eye find the curves delineating images by minimizing an energy involving area and

Received by the editors January 30, 1992 and, in revised form, February 1, 1993, and March 23, 1993.

1991 Mathematics Subject Classification. Primary 49Q20.

Key words and phrases. Area-minimizing, length of singular curves. 


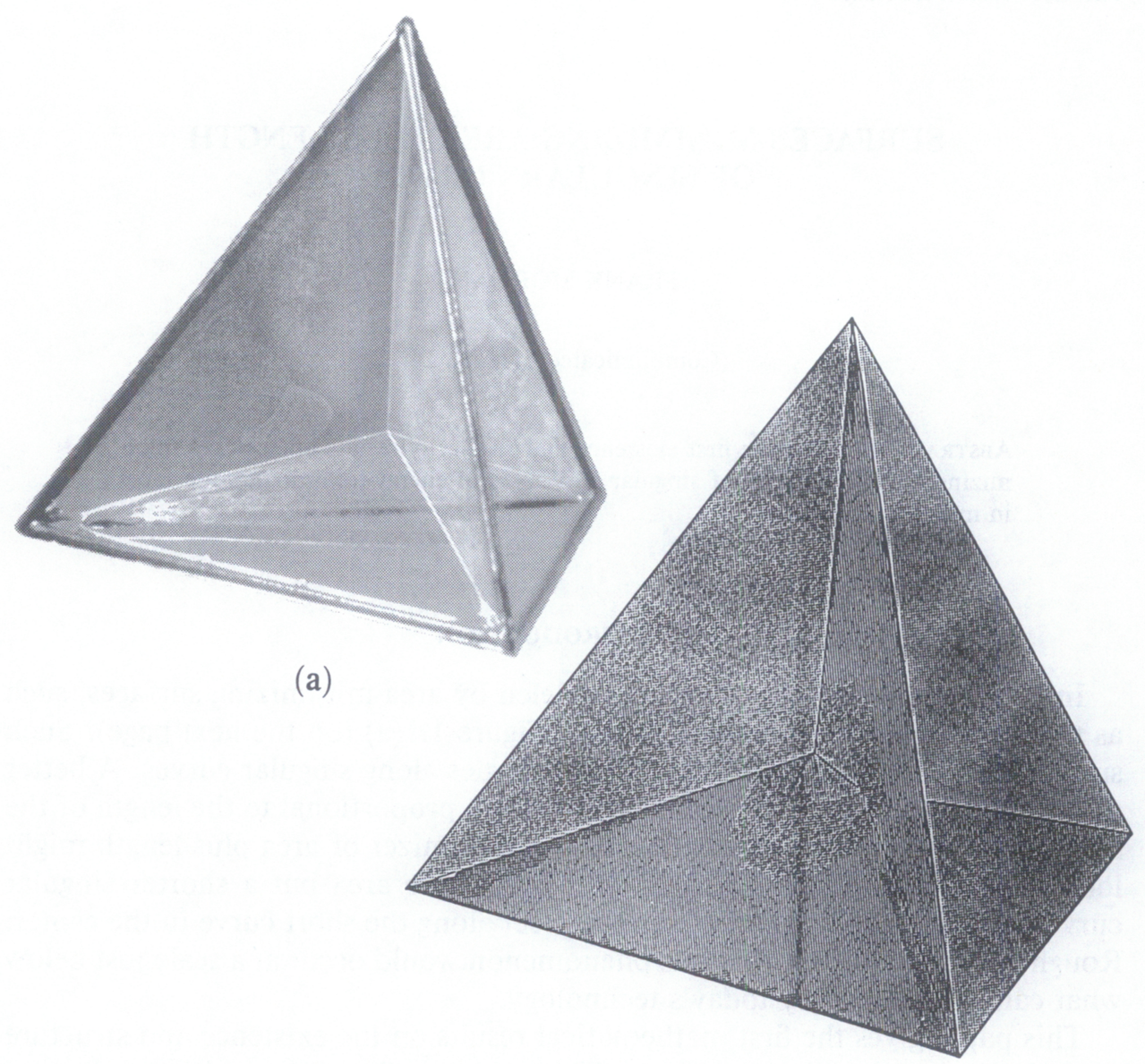

(b)

FIGURE 1.1. The area-minimizing soap film (a) models interfaces in materials. If an energy cost is added for the singular curves, surface (b) becomes cheaper than (a). Note that four pieces of surface meet along the short curve in the center. (Photo by F. Goro. Computer picture by J.Taylor.)

length in a planar domain. Our surfaces in space present completely different problems.

1.1. The main results. Following Brakke (cf. [Br1]), our existence and regularity results (Theorem 2.1) require an a priori prescription of the combinatorial structure of the solution, including the number of singular curves and which pieces of surface they bound. The theorem concludes that each singular curve $C_{i}-\partial C_{i}$ is smoothly embedded, that the associated pieces of surface are smooth minimal submanifolds with boundary along $C_{i}-\partial C_{i}$, and that their inward conormals $n_{1}, n_{2}, \ldots$ and the curvature vector $\kappa$ of the curve satisfy the balancing condition

$$
n_{1}+n_{2}+\cdots+\kappa=0
$$


Unfortunately, the theorem theoretically allows unanticipated intersections and crossings of surfaces impossible for interfaces between regions.

We conjecture that without any combinatorial prescriptions, the solution is a smoothly embedded stratified manifold: minimal surfaces meet in threes or fours along curves, which in turn meet only in threes at isolated points (see Conjecture 3.1). That surfaces meet in threes or fours is suggested by recent work of the Williams College "SMALL" undergraduate research Geometry Group [CCF] on the analogous lower-dimensional problem of networks minimizing length plus the number of added nodes or "Steiner points". In such networks segments sometimes meet in threes or fours but never in fives or more.

1.2. Surfaces prescribed to meet $\nu$ at a time. For surfaces meeting $\nu$ at a time for a fixed integer $\nu$, there is a simpler approach: minimize area $(S)+$ $\frac{1}{\nu}$ length $(\partial S-B)$ among integral currents with $\partial S \equiv B$ (modulo $\left.\nu\right)$. This boundary condition means that

$$
\partial S-B=\nu C
$$

for some "singular curve" $C$. Existence follows from the compactness theorem for surfaces modulo $\nu$ (see [M2, 11.1]). In this context, known regularity says that the surface is embedded.

1.3. The thread problem. Section 5 describes some implications for the problem of finding a least-area surface or soap film bounded by a piece of wire and a piece of thread.

1.4. Edge-length-minimizing polyhedra. Another question involving the length of singular curves goes back to Fejes-Toth [FT, p. 305]:

Find the polyhedron of unit volume of least total edge length.

Melzak [Me, (B), p. 567] conjectured the equilateral right triangular prism. The data from Berger [Be], as compiled in Table 1, compares some of the leading contenders.

Raising the stakes from the $1991 \mathrm{Mt}$. Holyoke Regional Geometry Institute, I now offer \$200 for a solution. Meanwhile, I offer a copy of my book [M2] for finding an example that beats the triangular prism or for proving that a minimizer exists (even among convex polyhedra). The problem with existence is that infinitely many short edges may appear in the limit.

TABLE 1

\begin{tabular}{|c|c|}
\hline Polyhedron (unit volume & Length \\
\hline Triangular prism & 11.896 \\
\hline Cube & 12.000 \\
\hline Tetrahedron & 12.238 \\
\hline Pentagonal prism & 12.518 \\
\hline Square pyramid & 12.814 \\
\hline Triangular dipyramid & 14.342 \\
\hline Dodecahedron & 15.217 \\
\hline Octahedron & 15.419 \\
\hline Icosahedron & 23.13 \\
\hline
\end{tabular}




\section{EXISTENCE AND REGULARITY THEOREM}

The following theorem, given fixed boundary curves $C_{1}, \ldots, C_{m}$, produces surfaces $S_{1}, \ldots, S_{m_{2}}$, free boundary curves $C_{m+1}, \ldots, C_{m_{1}}$, and points $P_{1}, \ldots, P_{m_{0}}$, meeting in prescribed ways, minimizing $\sum \operatorname{area}\left(S_{i}\right)+$ $\sum_{j>m}$ length $\left(C_{j}\right)$. "Surface" technically means flat chain modulo two, thus allowing singularities, nonorientability, and unrestricted topological type (see [M2, Chapters 11, 1, 4, 5]).

2.1. Theorem. Existence and regularity with prescribed combinatorial structure. In $\mathbf{R}^{3}$ let $C_{1}, \ldots, C_{m}$ be rectifiable curves. Fix integers $m_{0} \geq 0, m_{1} \geq$ $m, m_{2} \geq 0$. Let $I_{i}, J_{i}$ be fixed sets of integer indices. If there are any surfaces (flat chains modulo two) $S_{1}, \ldots, S_{m_{2}}$, rectifiable curves $C_{m+1}, \ldots, C_{m_{1}}$, and points $P_{1}, \ldots, P_{m_{0}}$ such that

$$
\begin{array}{cc}
\partial S_{i}=\sum_{j \in I_{i}} C_{j} & \left(1 \leq i \leq m_{2}\right), \\
\partial C_{i}=\sum_{j \in J_{i}} P_{j} & \left(1 \leq i \leq m_{1}\right)
\end{array}
$$

(modulo two), then they may be chosen to minimize

$$
\sum \operatorname{area}\left(S_{i}\right)+\sum_{i>m} \text { length }\left(C_{i}\right)
$$

Each $S_{i}-\bigcup_{j \leq m} C_{j}-\bigcup P_{j}$ is a smooth, embedded minimal surface on the interior. Each singular curve $C_{j}-\partial C_{j}(j>m)$ is a $C^{1,1}$ embedded curve. At any nonboundary point in a single $C_{j}$, if $j>m$, each $S_{i}$ is a smooth $\left(C^{\infty}\right)$ embedded manifold with boundary; the curvature of $C_{j}$ does not exceed the number of surfaces $S_{i}$ it bounds by prescription (1), and the inward conormals $n_{1}, n_{2}, \ldots$ of these surfaces and the curvature vector $\kappa$ of $C_{i}$ sum to 0 :

$$
n_{1}+n_{2}+\cdots+\kappa=0 \text {. }
$$

If $P_{j}$ bounds only free boundary curves by prescription (2), i.e., if $j \in J_{i}$ only if $i>m$, the onward unit tangents to these curves at $P_{j}$ sum to 0 .

2.2. Example. One interesting example consists of $m \geq 3$ half-discs $S_{i}$ bounded by $m$ semicircles $C_{i}$ and a common diameter $C_{m+1}$, with the conormals summing to 0 . (Here $m_{0}=2, m_{1}=m+1, m_{2}=m, I_{i}=\{i, m+1\}$, each $J_{i}=\{1,2\}$, and all the $C_{i}$ have the same endpoints $P_{1}, P_{2}$.) This example separately minimizes both $\sum \operatorname{area}\left(S_{i}\right)$ and of course length $C_{m+1}$. It minimizes area by a standard slicing argument (cf. [M2, Lemma 10.6]). The slice itself is stationary because the $m$ vectors by hypothesis sum to 0 . Since length is a convex function of the position of the point where the $m$ vectors meet, the slice is the length-minimizing way to connect the $m$ given points to a free point.

The surface on the tetrahedral frame of Figure 1.1(b) is presumably another example.

2.3. Remark. The theorem and proof immediately generalize to minimize

$$
\sum \lambda_{i} \operatorname{area}\left(S_{i}\right)+\sum_{i>m} \mu_{i} \text { length }\left(C_{i}\right)
$$


for positive weights $\lambda_{i}, \mu_{i}$. Of course, the terms in the balancing condition (4) need to carry the associated weights.

2.4. Proof of Theorem. Since area $\left(S_{i}\right)$, length $\left(C_{i}\right)$, length $\left(\partial S_{i}\right)$, and $\operatorname{card}\left(\partial C_{i}\right)$ are bounded by (3), (3), (1), and (2), respectively, the existence of a minimizing collection follows by compactness (see [M2, 11.1, 5.5, 5.6] or [F1, (4.2.17), p. 432]).

Of course, each $S_{i}$ must be area minimizing for its boundary, hence a smooth, embedded minimal surface ([R] or [F2]).

Suppose $p$ lies in a single $C_{j}$ (but not in $\partial C_{j}$ ) and $j>m$. A first variation argument shows that weakly the curvature of $C_{j}(j>m)$ is at most the number of surfaces it bounds by prescription (1):

$$
|\kappa| \leq \kappa_{j} \equiv \operatorname{card}\left\{i: i \in I_{j}\right\}
$$

Otherwise smooth deformations of $C_{j}$ decreasing length (leaving the surfaces alone), coupled with the addition of small patches of surface, would decrease area plus length. This argument depends on the fact that the solution is minimizing, not merely stationary.

Therefore, $C_{j}$ is a $C^{1,1}$ immersion with Lipschitz constant on the derivative of an arclength parameterization at most $\kappa_{j}$. Since the surfaces are unoriented, $C_{j}$ must be embedded by a simple comparison argument.

Since the surfaces are unoriented, each associated $S_{i}$ is a $C^{1, \alpha}$ manifold with boundary along $C_{j}$ at $p \quad(0<\alpha<1)$ ([All1, p. 523], proved in [All2, $\S 5])$. Furthermore, if $C_{j}$ is $C^{k, \alpha}$, so is each $S_{i}$, by the standard Schauder theory [GT, Theorem 6.19]. The vanishing of the first variation of (3) yields (4) (weakly, since $C_{j}$ is not yet proved $C^{2}$ ).

Now by (4), since the conormals are $C^{\alpha}, \kappa$ is $C^{\alpha}$; hence, $C_{j}$ is $C^{2, \alpha}$; hence, each $S_{i}$ is $C^{2, \alpha}$; hence, the conormals are $C^{1, \alpha}$; hence, $\kappa$ is $C^{1, \alpha}$; hence, $C_{j}$ is $C^{3, \alpha}$; and so on. We conclude that $C_{j}$ is $C^{\infty}$ and that the surfaces meet smoothly along $C_{j}$ at $p$.

The final balancing condition at the singular points is the standard equilibrium condition for length-minimization, which applies here because at a small scale the area effects are negligible.

\section{A Conjecture}

The following conjecture removes the a priori restrictions on the combinatorial structure of Theorem 2.1 and gives stronger regularity. Regions are prescribed outside the unit ball. Singular curves are allowed to escape penalty by moving off to the boundary, as perhaps the material attempts to form layers.

3.1. Conjecture. Existence and regularity. Let $R_{1}, \ldots, R_{m}$ be measurable partitioning subsets of $\mathbf{B}(0,2) \subset \mathbf{R}^{3}$ prescribed in $\mathbf{B}(0,2)-\mathbf{B}(0,1)$. Let $S$ denote the interface between regions, restricted to $\mathbf{B}(0,1)$; i.e., $S=\bigcup \partial \mathbf{R}_{i} \cap$ $\mathbf{B}(0,1)$. Then $R_{1}, \ldots, R_{m}$ may be chosen to minimize area $S+\mathscr{H}^{1}(\operatorname{sing} S)$. Here $\operatorname{reg} S$ and $\operatorname{sing} S$ denote the portions of $S$ inside the open unit ball where $S$ is or is not a $C^{1}$ embedded manifold. $\operatorname{Reg} S$ is a smooth $\left(C^{\infty}\right)$ minimal surface. Sing $S$ consists of smooth curves, along which three or four pieces of surface meet smoothly, in turn meeting smoothly in threes at $120^{\circ}$ at isolated points. Along the singular curves, the inward conormals $n_{i}$ and the curvature 
vector $\kappa$ of the singular curve sum to 0 :

$$
\sum n_{i}+\kappa=0
$$

At the singular points, all pieces of surface have a common tangent plane.

3.2. Remarks. For similar "soap film" problems minimizing area alone, existence follows by viewing the $R_{i}$ as integral currents of geometric measure theory and minimizing $\frac{1}{2} \sum \mathbf{M}\left(\partial R_{i}\right)$. Work of Taylor [T] and Almgren [Alm] shows that minimizers consist of smooth surfaces meeting in threes at $120^{\circ}$ angles along curves, which in turn meet in fours at angles of about $109^{\circ}$.

The evidence for 3.1 that surfaces meet in threes and fours includes the tetrahedral example of Figure 1.1 and the lower-dimensional fact that certain networks minimizing length plus the number of Steiner points meet in threes and fours [CCF]. Five or more surfaces meeting could be stable but not minimizing.

The singular curves meet in threes because length-minimizing networks meet in threes. At such a singular point, the three curves determine the plane tangent to the pieces of surface.

The proof seems beyond current technology. The main obstacle in proving the conjectured existence is that additional singular curves could appear in the limit; i.e., $\mathscr{H}^{1}(\operatorname{sing} S)$ may not be lower semicontinuous. The main difficulty in proving regularity is that neither area nor length alone is minimized. On a small scale the singular curve should be nearly minimal, unless shortening it would create other singular curves.

We conjecture similar existence and regularity for the harder problem of regions of prescribed volumes minimizing area plus singular length. See the upcoming [M1].

\section{HIGHER DIMENSION AND CODIMENSION}

The existence results of Theorem 2.1 generalize to collections of surfaces $S_{i}^{d}$ of dimension $0 \leq d \leq m$ in $\mathbf{R}^{n}$ minimizing

$$
\sum_{d=1}^{m} \sum_{i} \operatorname{area}\left(S_{i}^{d}\right) .
$$

The $m$-dimensional surfaces are area minimizing and hence embedded minimal surfaces (away from their boundaries) except for an interior singular set of dimension at most $m-2$ [F2, Theorem 2, p. 771]. The lower-dimensional surfaces are $(\mathbf{M}, \varepsilon, \delta)$-minimal and hence embedded $C^{1}$ manifolds almost everywhere [Alm, IV.13(6)].

Conjecture 3.1 extends to hypersurfaces $S$ in $\mathbf{R}^{n}$ minimizing

$$
\mathscr{H}^{n-1}(S)+\mathscr{H}^{n-2}(\operatorname{sing} S) .
$$

$\operatorname{Reg} S$ is an embedded minimal hypersurface. Sing $S$ consists of $(n-2)$ dimensional surfaces along which three or four minimal hypersurfaces meet, $(n-3)$-dimensional surfaces along which three $(n-2)$-dimensional surfaces meet, $(n-4)$-dimensional surfaces along which four $(n-3)$-dimensional surfaces meet, and lower-dimensional singular sets. (Sing $S$ itself should have soap-film-like singularities, which are not yet classified for films of dimension $n-2>2$. See [T, W1, W2].) 


\section{THE THREAD PROBLEM}

For convenience consider a single surface $S_{1}$. Any minimizer of

$$
\text { area } S_{1}+\sum_{j>m} \text { length } C_{j}
$$

as before (or more generally area $S_{1}+\lambda \sum_{j>m}$ length $C_{j}$ for some $\lambda>0$ ) is automatically a minimizer of area $S_{1}$ given length $C_{j}$. This second problem may be interpreted as attaching fixed lengths of movable thread to fixed boundary curves $C_{1}, \ldots, C_{m}$ and seeking the least-area surface ("soap film") with that partially free boundary. See Ecker [E] or, for the classical mapping setting, Alt [A], Hildebrandt [H1, H2], and Nitsche [N]. Our conclusion (4) implies that the free boundary is an embedded curve of constant curvature 1 (more generally $1 / \lambda)$; cf. [E, Proposition 2.7], whose requirement that the surface be oriented means that the free boundary curve need not be embedded.

A solution to the second "thread" problem of minimizing area $S_{1}$ given length $C_{j}$ need not be a solution to the first problem of minimizing area $S_{1}+$ $\lambda \sum$ length $C_{j}$ for any Lagrange multiplier $\lambda$. For example, take a single fixed boundary curve $C_{1}$ to be a large arc of a circle of curvature $1 / 2$ as in Figure 5.1. Taking the single free boundary curve $C_{1}$ to be a large arc of a circle of curvature 1 provides the unique solution to the thread problem for its length, but taking $C_{2}=C_{1}$ (and $S_{1}=\varnothing$ ) minimizes area $S_{1}+$ length $C_{2}$ (indeed minimizes area $S_{1}+\lambda$ length $C_{2}$ for all $\lambda \leq 2$ ).

Actual soap films have interior singularities that complicate the problem even for a single fixed boundary curve closed by a single thread. For a boundary curve that is $(3,1)$ torus "knot", there is a soap film consisting of three strips meeting at $120^{\circ}$ along a singular curve. If a piece of the boundary is replaced by a suitably long piece of thread, the center of the thread will bound two strips of surface, and its curvature, determined by 2.1(4), will vary. See Figure 5.2. This soap film could be modeled by a version of Theorem 2.1 using surfaces modulo three.

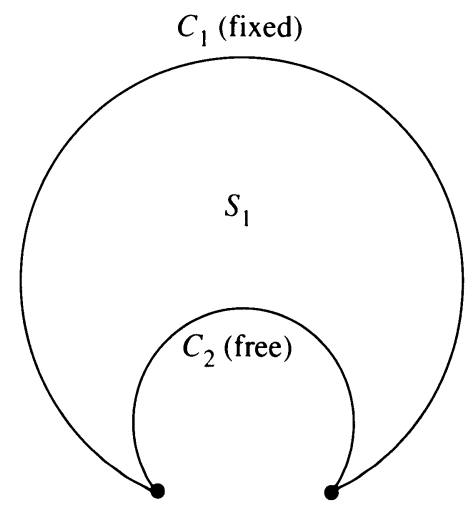

Figure 5.1. $C_{2}$ is the best thread for its length, but taking $C_{2}=C_{1}$ and $S_{1}=\varnothing$ minimizes area $S_{1}+$ length $C_{2}$. 


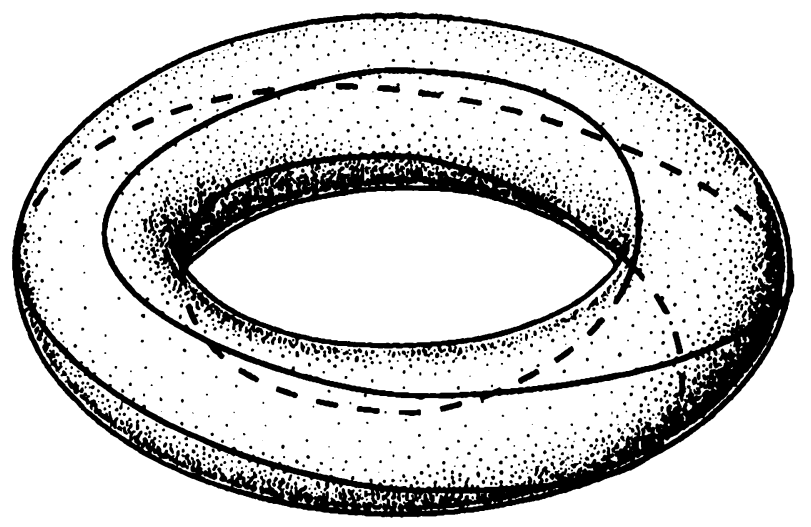

(a)

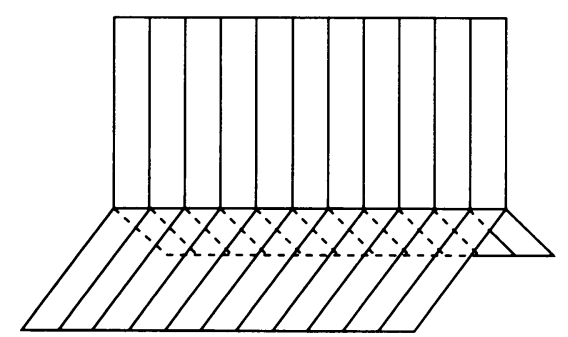

(b)

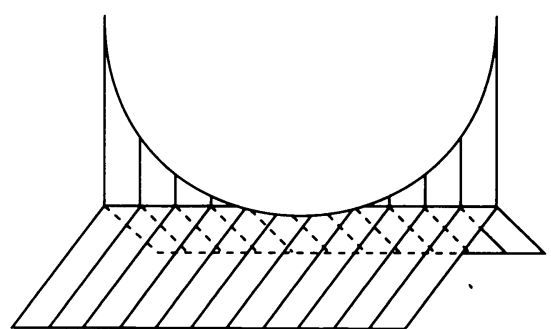

(c)

Figure 5.2. With actual soap films, if the thread extends through an interior singular curve, it will not have constant curvature. (b) shows a piece of a soap film bounded by the $(3,1)$ torus knot of $(a)$. A thread replacing a segment of boundary as in (c) will not have constant curvature.

\section{ACKNOWLEDGMENT}

The author thanks Ken Brakke for helpful conversations. This work was partially supported by an NSF grant and the Institute for Advanced Study.

\section{REFERENCES}

[Ab] Jeff Abrahamson, Curves length minimizing modulo $\nu$ in $\mathbf{R}^{n}$, Michigan Math. J. 35 (1988), 285-290.

[All1] William K. Allard, On boundary regularity for Plateau's problem, Bull Amer. Math. Soc. 75 (1969), 522-523.

[All2] On the first variation of a varifold: Boundary behavior, Ann. of Math. (2) 101 (1975), 418-446.

[Alm] F. J. Almgren, Jr., Existence and regularity almost everywhere of solutions to elliptic variational problems with constraints, Mem. Amer. Math. Soc., vol. 4, No. 165, Amer. Math. Soc., Providence, RI, 1976. 
[Alt] Hans Wilhelm Alt, Die Existenz eines Minimalfläche mit freimem Rand vorgeschriebrener Länge, Arch. Rational Mech. Anal. 51 (1973), 304-320.

[Be] Scott Berger, The search for boundary-minimizing polytopes in two and three dimensions, Junior Paper, Princeton University, Princeton, NJ, June 1991.

[Br1] Kenneth A. Brakke, Minimal cones on hypercubes, J. Geom. Anal. 1 (1991), 329-338.

[Br2] - The surface evolver, Experiment. Math. 1 (1992), 141-165.

[CCF] T. Colthurst, C. Cox, J. Foisy, H. Howards, K. Kollett, H. Lowy, and S. Root, Networks minimizing length plus the number of Steiner points, Williams College SMALL Undergraduate Research Geometry Group, Network Optimization Problems: Algorithms, Complexity and Applications (Dingzhu Du and Panos M. Pardalos, eds.), World Scientific, 1993, pp. 23-26.

[C] Edith A. Cook, Free boundary regularity for surfaces minimizing $\operatorname{Area}(S)+c \operatorname{area}(\partial S)$, Trans. Amer. Math. Soc. 290 (1985), 503-526.

[DG] E. De Giorgi, Su alcuni problemi comuni all'analisi e alla geometria, Note Mat. IX-Suppl. (1989), 59-71.

[E] Klaus Ecker, Area-minimizing integral currents with movable boundary parts of prescribed mass, Ann. Inst. H. Poincaré 6 (1989), 261-293.

[F1] Herbert Federer, Geometric measure theory, Springer-Verlag, New York, 1969.

[F2] - The singular sets of area minimizing rectifiable currents with codimension one and of area minimizing flat chains modulo two with arbitrary codimension, Bull. Amer. Math. Soc. 76 (1970), 767-771.

[FT] L. Fejes-Toth, Regular figures, Macmillan, New York, 1964.

[GT] David Gilbarg and Neil S. Trudinger, Elliptic partial differential equations of second order, Springer-Verlag, New York, 1983.

[H1] S. Hildebrandt, Ein einfacher Beweis für die Regularität der Lösungen gewisser zwei dimensionaler Variations probleme unter freien Randbedingungen, Math. Ann. 194 (1971), 316-331.

[H2] _ On two isoperimetric problems with free boundary conditions, Variational Methods for Free Surface Interfaces (Paul Concus and Robert Finn, eds.), Springer-Verlag, New York, 1986, pp. 43-51.

[LM] Gary Lawlor and Frank Morgan, Paired calibrations applied to soap films, immiscible fluids, and surfaces or networks minimizing other norms, Pacific J. Math. (to appear).

[Me] Z. A. Melzak, Problems connected with convexity, Canad. Math. J. 8 (1965), 565-573.

[M1] Frank Morgan, Clusters minimizing area plus length of singular curves, Math. Ann. (to appear).

[M2] — Geometric measure theory: a beginner's guide, Academic Press, New York, 1988.

[MT] Frank Morgan and Jean Taylor, Destabilization of the tetrahedral point junction by positive triple junction line energy, Scripta Met. Mat. 25 (1991), 1907-1910.

[MuS] David Mumford and Jayant Shah, Optimal approximation by piecewise smooth functions and associated variational problems, Comm. Pure Appl. Math. 42 (1989), 577-685.

[N] Johannes C. C. Nitsche, The regularity of minimal surfaces on the movable parts of their boundaries, Indiana Univ. Math. J. 21 (1971), 505-513.

[R] E. R. Reifenberg, Solution of the Plateau problem for m-dimensional surfaces of varying topological type, Acta Math. 104 (1960), 1-82.

[T] Jean Taylor, The structure of singularities in soap-bubble-like and soap-film-like minimal surfaces, Ann. of Math. (2) 103 (1976), 489-539.

[W1] Brian White, Regularity of the singular sets in immiscible fluid interfaces and in solutions to other plateau-type problems, Proc. Centre Math. Anal., Austral. Nat. Univ., Canberra, 1985, pp. 244-249.

[W2] _ Regularity of singular sets for plateau-type problems (in preparation).

Department of Mathematics, Williams College, Williamstown, Massachusetts 01267

E-mail address: Frank.Morgan@williams.edu 